\section{Our students, our} future

Every reader of the Canadian Journal of Public Health will likely know at least one student, whether undergraduate or graduate, who is working towards a career in public health or learning new skills to enhance their current competencies and career options. Among them are those who will be our future leaders.

Now is an auspicious time to be a student in public health. There is renewed intention across Canada to define educational and service standards for public health and to declare common core competencies for high-quality professional practice. An imminent national consultation about schools of public health provides a timely opportunity to compare models and take stock of current and future capacity needs. As in many other professional curriculae, students learn invaluable lessons from exposure to practitioners in service and policy before graduation. Guidelines for Masters of Public Health programs specify the importance of practica for this purpose. Students acquire research skills through the experience of planning, conducting and writing up their own projects. Effective leadership and management skills in public health can be effectively honed through feedback, mentoring and access to relevant programs.

At any point of a career in public health, access to evidence is essential. To strengthen the scientific basis of our field, the importance of a prestigious journal with a network of willing and expert peer reviewers cannot be underestimated. Yet how do we equip our students with the necessary skills and judgement to become constructive and discerning peer reviewers?

Solutions have come from the students themselves. The editorial office recently welcomed the creation of the Association's first-ever Student Editorial Group. This Group asks faculty members in universities and service-based research groups to renew their efforts to impart the skills of peer review. Consider the benefits when a manuscript is reviewed by a group of students, supported in this process by a supervisor within their academic department to tutor them in peer review. At the Journal, we provide reviewers with structured review forms according to study design. Imagine the palpable 'buzz' within a group of students engaged in using such a tool and learning at the same time about research methods, manuscript preparation and public health significance. We will soon ask authors to indicate in their covering letter whether they would prefer to 'opt-out' from such a proposal. Those who 'opt-in' will be augmenting the wealth of opportunities whereby students learn their craft.

Jeanette Ward, MBBS, MHPEd, PhD, FAFPHM

Interim Scientific Editor, CJPH
LE MOT DE LA RÉDACTION

Nos étudiants, notre avenir

Tous les lecteurs de la Revue canadienne de santé publique connaissent sans doute au moins une étudiante ou un étudiant, diplômé ou de premier cycle, qui veut faire carrière dans la santé publique ou qui se lance dans ce domaine pour compléter sa formation ou élargir ses possibilités d'emploi. Parmi ces étudiants se trouvent nos leaders de demain.

Le moment est favorable aux études en santé publique. Partout au Canada, on se promet à nouveau de définir des normes d'enseignement et de services et d'instaurer des compétences de base communes pour que les usages de la profession soient de haute qualité. Une consultation pancanadienne à venir bientôt au sujet des écoles de santé publique est l'occasion idéale de comparer des modèles et de faire le point sur nos besoins d'effectifs actuels et futurs. Comme dans bien d'autres domaines d'études professionnelles, les étudiants apprennent de précieuses leçons en étant exposés, avant d'obtenir leur diplôme, à des praticiens qui offrent des services ou qui élaborent des politiques. Dans les lignes directrices des programmes de maîtrise en santé publique, on souligne l'importance des stages à cet égard. C'est en planifiant, en exécutant et en présentant leurs propres projets que les étudiants deviennent de bons chercheurs. Et c'est par la rétroaction, l'encadrement et l'accès aux programmes pertinents qu'ils perfectionnent leurs compétences de leadership et de gestion.

À toute étape d'une carrière en santé publique, il est essentiel d'avoir accès à des données probantes. Pour renforcer les fondements scientifiques de notre domaine, on ne saurait sous-estimer l'importance d'une revue prestigieuse à laquelle collabore tout un réseau d'évaluateurs de textes chevronnés et de bonne volonté. Mais comment inculquer à nos étudiants les compétences et le jugement nécessaires pour qu'ils deviennent des évaluateurs utiles et perspicaces?

Les étudiants eux-mêmes nous offrent la clé du problème. Le bureau de la rédaction a salué récemment la création du tout premier comité de rédaction étudiant de l'Association. Ce comité demande aux professeurs d'université et aux groupes de recherche axés sur les services de redoubler d'efforts pour enseigner les compétences d'évaluation par les pairs. Songez aux avantages de faire évaluer un manuscrit par un groupe d'étudiants, avec l'aide et sous la supervision d'un professeur du département qui leur apprendra les rudiments de l'évaluation par les pairs. Ici à la Revue, nous offrons à nos évaluateurs des fiches d'évaluation structurées selon le plan d'étude des manuscrits. Imaginez un peu le bourdonnement palpable d'un groupe d'étudiants en train d'utiliser un tel outil tout en apprenant les méthodes de recherche, la préparation des manuscrits et l'importance de la santé publique. Nous demanderons bientôt à nos auteurs d'indiquer dans leur lettre d'accompagnement s'ils préferent ne pas participer à une telle démarche. Ceux qui choisiront plutôt d' "embarquer " élargiront les possibilités qu'ont les étudiantes et les étudiants d'apprendre leur métier.

Jeanette Ward, MBBS, MHPEd, Ph.D., FAFPHM

Rédactrice-réviseure scientifique intérimaire de la RCSP 